\title{
Endoscopic ultrasound-guided colonic anastomosis: pushing the boundaries
}

Lumen-apposing metal stents (LAMS) have pushed the boundaries of interventional EUS beyond drainage of pancreatic fluid collections. EUS-guided gastroenterostomy (EUS-GE) using LAMS is now an accepted treatment modality for malignant gastric outlet obstruction (GOO) at many institutions. EUS-GE has multiple advantages over enteral stenting, including decreased risk of recurrent GOO due to stent occlusion. EUS-GE entails formation of an anastomosis between the stomach and a small bowel segment distal to the site of obstruction, usually the distal duodenum or proximal jejunum.

EUS-guided anastomosis formation has also been described at other sites, such as duodenojejunostomy (EUS-DJ) and jejunojejunostomy (EUS-JJ). EUS-DJ can be utilized in patients with malignant distal duodenal or proximal jejunal obstruction when EUS-GE is not possible, or for facilitation of subsequent endoscopic retrograde cholangiopancreatography (ERCP) in patients with difficult surgical anatomy and in whom standard ERCP has failed. EUS-JJ can be employed to treat afferent or efferent loop obstructions in patients with surgically altered anatomy (e. g., after pancreaticoduodenectomy). Despite the multiple routes and access points for EUS-guided anastomosis creation described above, interventional endosonographers have predominantly avoided the colon. This is likely multifactorial, but reasons include fear of fecal leakage, perforation, and peritonitis. The colon is probably an easier target for EUS-guided anastomosis than is the jejunum, simply because of its larger lumen. The cecum/ascending colon and transverse colon are in proximity to the duodenum and stomach, respectively. Sooklal and Kumar reported a case of a 43-yearold man with inoperable malignant small bowel obstruction (SBO) in the mid-ileum [1]. A percutaneous venting tube was not possible due to peritoneal carcinomatosis. EUS-guided duodeno-
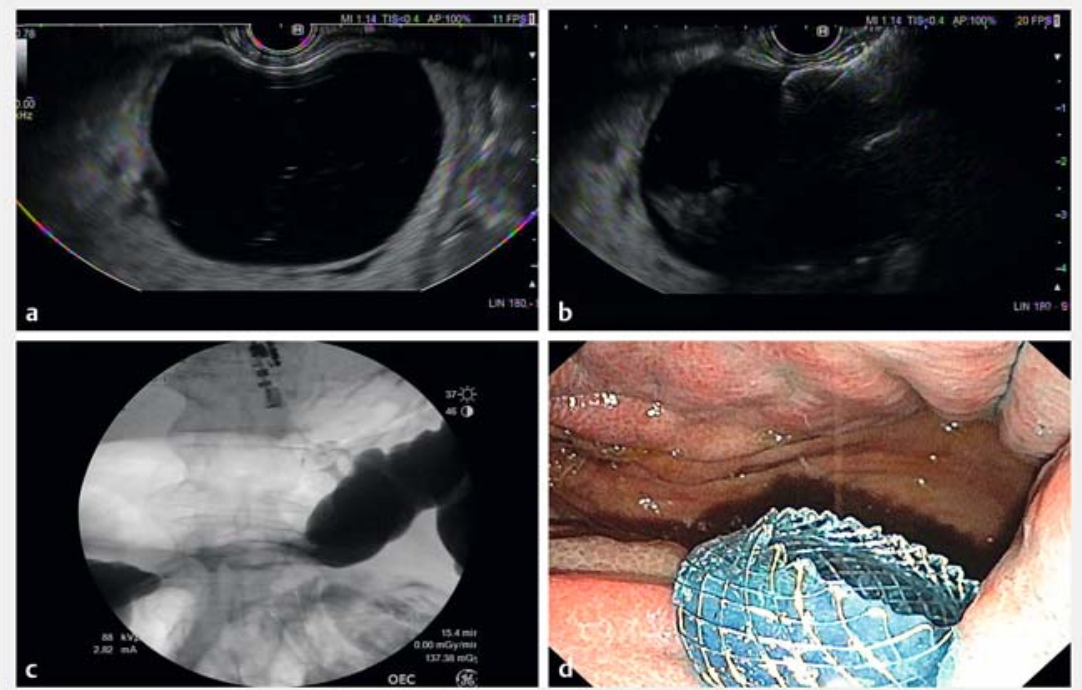

- Fig. 1 Endoscopic ultrasound (EUS)-guided gastrocolostomy in a patient with inoperable multilevel malignant small bowel obstruction. a Sonographic view of dilated transverse colon loop seen in apposition to the stomach. b This loop was initially punctured with a 19-gauge fine-needle-aspiration needle and blue dye was aspirated, confirming colonic access. EUSguided gastrocolostomy was then performed using a freehand technique with placement of a $15 \mathrm{~mm} \times 15 \mathrm{~mm}$ lumen-apposing metal stent. c Radiographic view of the deployed stent. d Endoscopic view of the deployed stent. Flow of blue-tinged dye through the lumen of the stent was seen.

colostomy (EUS-DC) was performed between the second duodenum and the cecum after initially advancing a colonoscope to the cecum and filling it with fluid. Nausea and vomiting resolved and the patient was able to tolerate comfort food by mouth. Mir and colleagues reported the case of a 72-year-old woman with inoperable metastatic cancer and history of colonic stent placement, who presented with malignant SBO [2]. An echoendoscope was advanced transanally and an ileocolonic anastomosis was created using a LAMS between the sigmoid colon and ileum, allowing resumption of tube feeding. No adverse events occurred in either of these two cases, and both patients ended up under hospice care due to their end-stage malignant disease.

These two cases highlight the feasibility of EUS-guided enterocolonic (EUS-EC) anastomoses using LAMS. We recently encountered a 64-year-old man with a history of appendiceal cancer treated with surgical resection and ileocolonic anastomosis. He later presented with metastasis and symptomatic multilevel SBO in the distal duodenum, jejunum, and ileocolonic anastomosis. Symptoms included abdominal pain, distention, intolerance to oral intake, nausea, and vomiting. The patient desired to consume comfort food orally and refused a decompression percutaneous endoscopic gastrostomy (PEG) tube. An EUS-guided approach was discussed with the patient and the multidisciplinary team. EUS-DC and EUS-guided gastrocolostomy (EUSGC) were deliberated as options to bypass and relieve symptoms of multilevel SBO. Potential risks of perforation, fecal peritonitis, diarrhea, and malnutrition were discussed. Colonoscopy was performed 
using an adult colonoscope after the coIon was cleansed with multiple enemas. About $500 \mathrm{~mL}$ of fluid (saline, contrast, and methylene blue) was infused in the right colon. The colonoscope was withdrawn and a linear echoendoscope was advanced transorally. The intention was to target a right colonic loop to minimize the risk of diarrhea. A well-distended and fluid-filled transverse colon loop in proximity to the gastric wall was located sonographically and radiographically. This was punctured with a 19-gauge needle under EUS guidance with aspiration of bluetinged fluid confirming colonic access. A $15 \mathrm{~mm} \times 15 \mathrm{~mm}$ LAMS (Hot Axios; Boston Scientific, Marlborough, Massachusetts, USA) was then placed under EUS guidance using the freehand technique, forming a gastrocolostomy ( $\triangleright$ Fig. 1 ; $\triangleright$ Video 1). The procedure was completed without any adverse events. The patient was placed on a twice-daily proton pump inhibitor (PPI) and started on a liquid diet and total parenteral nutrition (TPN). All obstructive symptoms resolved and he was discharged home on a full liquid diet 9 days after the procedure. At 6 weeks after the procedure, he reported eating semisolid food without nausea, vomiting, or diarrhea. He had discontinued his TPN at his own discretion and has been able to maintain his weight.

The three cases discussed above illustrate scenarios where EUS-guided gastrojejunostomy (EUS-GJ) or enteral stenting are not feasible options to alleviate symptoms of complex SBO, necessitating an EUS-EC or EUS-GC approach to bypass the obstruction and allow some degree of oral intake. These cases suggest the feasibility and safety of both approaches, recognizing the colon as a possible target organ for EUS-guided anastomoses in highly selected cases. Below are salient discussion points relevant to this emerging field.

\section{Selection of cases}

Enteral stenting and EUS-GJ remain the two major endoscopic methods for palliation of malignant GOO. EUS-DC/GC should only be considered for inoperable patients with more complex/distal obstruction where the standard techniques are not possible. Standard management

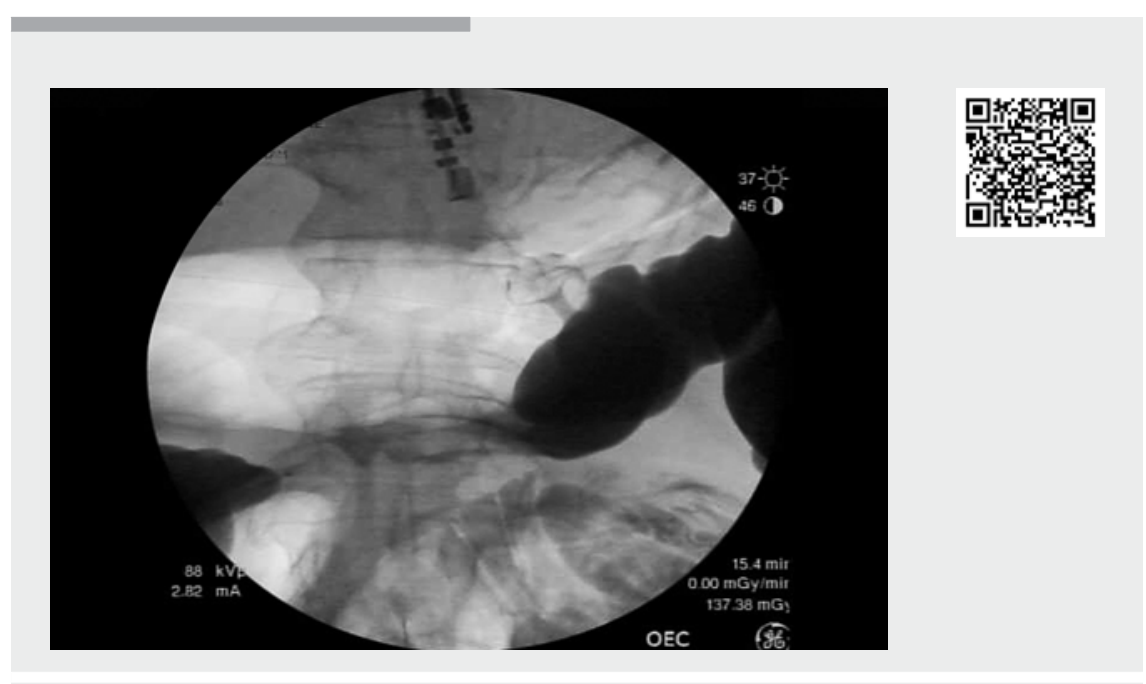

Video 1 Endoscopic ultrasound-guided gastrocolostomy procedure.

of patients with more distal obstruction is via venting PEG and TPN. The advantage of EUS-DC/GC is allowance of some oral intake, which can be valued by these terminal patients.

\section{Technique}

We follow the technique previously described for EUS-G]. A crucial step is generous filling of the colon with fluid for distention and apposition to the stomach/duodenum. We add methylene blue to the fluid mixture, which aids in correct location of the target as mentioned above. As with EUS-G], a freehand method is advisable during EUS-DC/GC. Advancement of a wire creates a false sense of security and tends to push the target organ (e.g., colon) away from the stomach/duodenum, risking stent misdeployment.

\section{Choice of access point}

A transoral approach with either a transgastric or transduodenal access to the colon is a feasible technique to create, respectively, a gastrocolostomy or a duodenocolostomy. It is unknown if the access route has an effect on outcomes; however, in our opinion the choice of the access point should be based on the shortest distance to the colon while maintaining a stable and straight echoendoscope if possible. Careful review of transabdominal images is helpful in determining the most appropriate technique.

\section{Alternative approaches}

A transanal approach for the creation of colonic anastomosis may be feasible in select cases, as illustrated by Mir and colleagues, who created an enterocolostomy between the ileum and the sigmoid colon. A transanal approach with enterocolonic anastomosis between the right colon and ileum would also be feasible in patients without obstructive colonic pathology; however, a forward-viewing echoendoscope may be needed in some cases.

\section{Lessons learned from the surgical literature}

The vast majority of reported cases represent surgical errors where either a gastroileostomy or gastrocolostomy is created inadvertently [3]. In these cases patients can develop diarrhea, dumping syndrome, colonic ulceration, weight loss, and nutritional deficiencies. On the other hand, one case report described a surgical duodenocolostomy technique to treat a patient with massive mesenteric infarction after aortic surgery [4]. This procedure allowed resumption of normal oral intake with long-term survival. Another case report described long-term survival 
of a patient with massive mesenteric infarction treated with mid-gut resection and duodenocolostomy [5].

The following are further lessons learned from the surgical literature:

1. Close monitoring of patients for the above-mentioned complications is warranted. Diarrhea can be managed with antidiarrheal medications and/or codeine. We initially placed our patient on octreotide to diminish pancreaticobiliary output; however, this was discontinued. In fact, he required laxatives for narcotic-induced constipation.

2. Periodic monitoring of nutritional parameters (e.g., albumin) and electrolytes is warranted.

3. PPIs should be prescribed to avoid colonic ulceration.

4. It is important to be aware that vomiting can occur hours after food intake, likely due to coloduodenal or cologastric reflux.

5. Although a gastrocolostomy or duodenocolostomy may allow adequate oral intake, it is crucial to understand that the purpose of a gastrocolostomy or duodenocolostomy is symptom palliation and allowance of oral intake for comfort. It is expected that these patients will be TPN-dependent and thus should be followed by nutrition specialists and treated similarly to patients with short bowel syndrome.

Endoscopy_UCTN_Code_TTT_1AS_2AG
Competing interests

Mouen A. Khashab is a consultant for Boston Scientific, Olympus, Medtronic, and GI Supply, and receives royalties from UpToDate and Elsevier.

The authors

\section{Sarah S. Al Ghamdi, Mouen A. Khashab}

Division of Gastroenterology and Hepatology, Johns Hopkins Hospital, Baltimore, Maryland, USA

\section{Corresponding author}

Mouen A. Khashab, MD

Division of Gastroenterology and Hepatology, Zayed Building, Suite 7125G, Johns Hopkins Hospital, 1800 Orleans Street, Baltimore, MD 21287, USA

mkhasha1@jhmi.edu

\section{References}

[1] Sooklal S, Kumar A. EUS-guided enterocolostomy for palliation of malignant distal small-bowel obstruction. VideoGIE [Internet] 2019; 4: 530-531

[2] Mir A, Parekh PJ, Shakhatreh M et al. Endoscopic ultrasound-guided creation of an enterocolostomy to relieve malignant bowel obstruction. Endosc Int Open 2019; 07: E1034-E1037

[3] Michels AG, Brown CH, Crile G]r. Surgical error of gastroileostomy: report of six cases. Am J Surg 1951; 82: 191-197
[4] Williams IM, Davies C] et al. Survival after resection and duodenocolostomy. Ann R Coll Surg Engl 1996; 78: 69-70

[5] She ZF, Yang XF, Ma L et al. Survival of massive mesenteric infarction through midgut resection and duodenocolostomy - a case report. Int J Colorectal Dis 2016; 31: 159160

\section{Bibliography}

Endoscopy 2021; 53: E367-E369

DOI $10.1055 / \mathrm{a}-1293-6483$

ISSN 0013-726X

published online 26.11.2020

(c) 2020. Thieme. All rights reserved.

Georg Thieme Verlag KG, Rüdigerstraße 14,

70469 Stuttgart, Germany

\section{ENDOSCOPY E-VIDEOS}

https:|/eref.thieme.de/e-videos

回的回 Endoscopy E-Videos is a free access online section, reporting 靣: on interesting cases and new techniques in gastroenterological endoscopy. All papers include a high quality video and all contributions are freely accessible online.

This section has its own submission website at https://mc.manuscriptcentral.com/e-videos 